\title{
Pseudoelastic behaviour of perforated NiTi shape memory plates under tension
}

\author{
Bashir S. Shariat ${ }^{\mathrm{a}}$, Yinong Liu ${ }^{\mathrm{a}}$ and Gerard Rio ${ }^{\mathrm{b}}$ \\ ${ }^{a}$ Laboratory for Functional Materials, School of Mechanical and Chemical Engineering, The \\ University of Western Australia, Crawley, WA 6009, Australia \\ Tel: +61 8 64883132, Fax: +61 8 64881024, email: yinong.liu@uwa.edu.au \\ bLaboratoire d’Ingénierie des Matériaux de Bretagne, Université de Bretagne Sud, Université \\ Européenne de Bretagne, BP 92116, 56321 Lorient cedex, France
}

\begin{abstract}
This paper reports the tensile deformation behaviour of near-equiatomic NiTi plates with circular, elliptical and square holes. The investigation is done both experimentally and by mathematical modelling. It is found that the nominal stress-strain curve of such structures deviates from typical stress-strain variation of NiTi with flat stress plateaus, by exhibiting stress gradients over the forward and reverse transformations of the hole-affected areas. The hole-affected length is nearly triple the lateral dimension of the hole, and when $33 \%$ of the gauge length is covered by (circular) holes, the entire sample behaves like a functionally graded material. Such mechanical behaviour is advantageous for achieving better loaddisplacement controllability and wider stress window for shape memory actuation and sensing.
\end{abstract}

Keywords: A. intermetallics, miscellaneous; B. martensitic transformations; B. mechanical properties at ambient temperature; B. shape-memory effects; F. mechanical testing, G. shapememory alloy applications

\section{Introduction}

Near-equiatomic NiTi alloy deforms via stress-induced martensitic transformation [1], which exhibits hystoelastic mechanical behaviour with large recoverable nonlinear deformation, known as the pseudoelasticity [2]. The pseudoelasticity of NiTi often manifests in a Lüderstype manner over a stress plateau [3]. The stress plateau level highly depends on heat treatment condition [4].This unique property has facilitated many engineering applications. In most applications, NiTi is used in thin wire forms under tensile loading [5, 6], to benefit from the high force output in tension and the rapid actuation due to fast thermal conduction. Other common forms include helical springs and thin walled tubes. However, some applications require the use of more complex shapes and under more complex loading conditions, such as perforated plates [7] or holed structures [8], NiTi thin films [9, 10], functionally graded NiTi structures [11-13], cellular structures [14] or surgical stents [15], woven NiTi layers embedded in composite structures [16], and porous NiTi [17, 18]. The complex geometry alters the mechanics conditions, thus mechanical behaviour of the components. For such conditions, standard uniaxial tensile testing of straight forms (e.g., wires and strips) is inadequate to describe or predict the mechanical behaviour of the component. This study deals with the pseudoelastic behaviour of perforated NiTi plate under tension.

Perforated plates are generally used as heat exchangers [19], sound absorbers [20], screens and filters [21]. The perforated or porous SMAs, in particular, can be used as orthopaedic devices, such as artificial bone implants, spinal vertebrae spacers [22] and skull plates (fixation plates for craniotomy operation) [15]. The existence of hole(s) in a SMA plate can be considered a geometrical defect to provoke transformation localisation [23]. Because of 
variations in geometry, different locations in a holey plate experience variations in stress state. This inhomogeneous stress field induces local martensitic transformation in the structure at different loading levels, complicating the global deformation behaviour of the plate $[24,25]$. Some recent finite element studies have investigated the combined effect of transformation and elasticity on pseudoelastic response of NiTi plates with arrays of holes $[26,27]$.

Perforated plates also provide a study case as a 2D model for porous structures. The deformation behaviour of porous SMA structures has been mainly studied under compression [28-33]. The effect of porosity on global stress-strain variation has been discussed. The numerical modellings of such structures mostly implement micromechanical averaging techniques or periodic patterns of pores [34]. In this article, we explore the effect of holes on deformation behaviour of perforated $\mathrm{NiTi}$ plates (strips) under tension. Mathematical expressions are derived to describe the nominal stress-strain variation of such components.

\section{Experimental investigation}

Commercial Ti-50.8at\%Ni sheets of $0.1 \mathrm{~mm}$ thickness supplied by Memry were used in this study. The sheets were in annealed state with acid pickled, oxide-free white surface finish. The perforated plate samples were prepared by electric discharge machining. The samples prepared by this method maintain excellent surface condition with minimal stress concentrations due to non-mechanical contact fabrication process. The tensile experiments were carried out at the strain rate of $2.4 \times 10^{-4} / \mathrm{s}$ and $303 \mathrm{~K}$ with fanned air cooling to maintain the isothermal condition. For each deformation test a new sample was used. Fig. 1 presents the stress-strain diagram of a uniform strip $(4 \mathrm{~mm} \times 30 \mathrm{~mm})$ of such material under tension. Partial deformation cycles were carried out. The transformation properties and elastic moduli are defined on the stress-strain curve using red dash lines. The material exhibited good pseudoelastic behaviour, with a full deformation recovery of up to $8 \%$, which is $\sim 2 \%$ beyond the end of the stress plateau. The critical stress at $303 \mathrm{~K}$ for the forward $\mathrm{A} \rightarrow \mathrm{M}$ transformation is $\sim 380 \mathrm{MPa}$ and that for the reverse $\mathrm{M} \rightarrow \mathrm{A}$ transformation is $\sim 140 \mathrm{MPa}$. Here, $\mathrm{A}$ and $\mathrm{M}$ refer to the austenite and martensite phases, respectively. Also shown in this figure is the thermal transformation behaviour of the alloy as measured by differential scanning calorimetry.

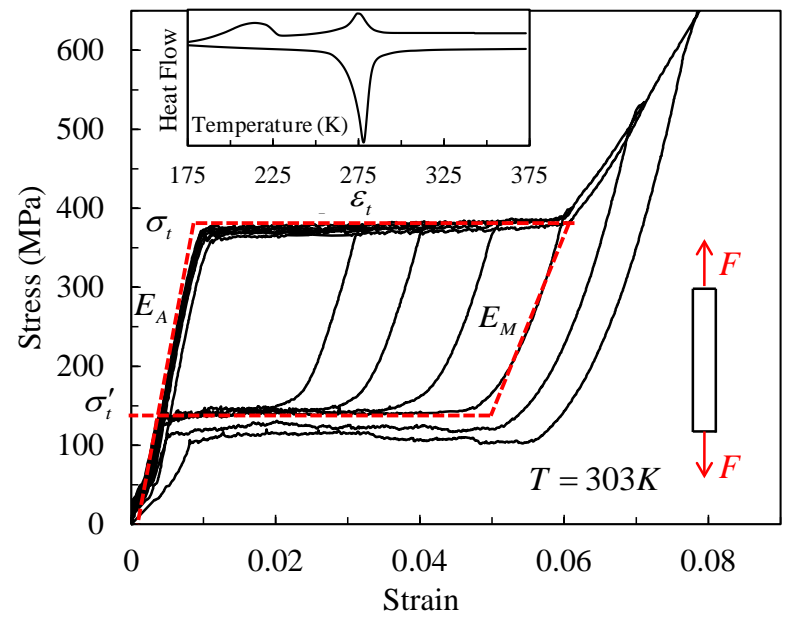

Fig. 1. Thermomechanical properties of the Ti-50.8at\%Ni alloy

Fig. 2 shows the deformation behaviour of NiTi plates of $9 \mathrm{~mm} \times 35 \mathrm{~mm}$ (gauge section) with circular holes. The holes were created symmetrically along the loading axis of the 
samples. Figs. 2(a), (b) and (c) show the nominal stress-strain variations of the samples with one, two and three holes of $3 \mathrm{~mm}$ in diameter, respectively. The distance between the centres of the neighbouring holes is $16 \mathrm{~mm}$ for the sample with two holes and $12 \mathrm{~mm}$ for the sample with three holes. The nominal stress is defined as the axial load $F$ divided by the initial full cross-sectional area of the plate (at where without holes), and the nominal strain is calculated as the total elongation of the plate $\Delta L_{\text {Tot }}$ divided by the initial length $L$ [35]:

$$
\sigma=\frac{F}{b h}, \quad \varepsilon=\frac{\Delta L_{T o t}}{L}
$$

where $b$ and $h$ are the width and the thickness of the plate, respectively. All samples exhibit good pseudoelastic behaviour. However, the nominal stress-strain curves over $\mathrm{A} \leftrightarrow \mathrm{M}$ transformations deviated from the flat stress plateaus observed in Fig. 1. Due to geometrical heterogeneity caused by the holes, the samples experienced normal stress variation across the loading direction in the course of tensile loading. The cross-sectional area is smaller where there are holes, therefore the stress is larger and the plate begins to transform ahead of the monolithic transformation in Fig. 1 at lower nominal stress defined by Eq. (1). The $A \rightarrow M$ transformation initiated in the regions at the sides of the holes, where the cross-sectional area is the smallest and the real stress is the highest, and gradually propagated into regions of higher cross sections as the load increases. Because there is a gradient in the cross-sectional area as it approaches the nominal cross-sectional area $(b h)$, there is also a gradient to the stress-strain curve in this region. As the transformation fronts reached to the solid part of the plate (with cross-sectional area $b h$ ), the nominal stress remained relatively constant until the entire structure was transformed to martensite.

The stress variation during stress-induced martensitic transformation may be divided into two regimes, as indicated in Fig. 2(a). Following the discussion of the relationship between crosssectional area and nominal stress given in the previous paragraph, the first regime is related to the section of the strip sample affected by the hole(s) and the second regime is related to the solid section(s) of the strip sample. Taking sample (a) for example, the length ratio between the hole section (the diameter of the hole in the length direction) and the total gauge length is $\eta_{h}=0.09$, whereas the ratio of the transformation strain of regime I $\left(\varepsilon^{I}\right)$ to the total transformation strain $\left(\varepsilon^{t}\right)$ is $\eta_{s}=0.27$. It is seen that $\eta_{h}<\eta_{s}$. This implies that the effect of the hole expanded beyond the diameter of the hole, as schematically indicated by the shaded section in sample (a) in Fig. 2(a). With the same argument, the hole-affected lengths are also marked in samples (b) and (c). It is seen that the hole affected length is approximately triple the diameter of the hole and that when hole diameter covers $33 \%$ of the gauge length, the entire sample behaves like a functionally graded material [36]. This information is useful for material design for functionally graded NiTi components.

Fig. 2(d) replots the full deformation cycles of the three perforated NiTi plate samples together in addition to that of a solid plate sample. It is seen that the forward transformation started at practically the same value of nominal stress ( 250 MPa). This loading level provided mean local stress of $\sim 380 \mathrm{MPa}$ at the minimum cross-sectional areas of the plates to induce $\mathrm{A} \rightarrow \mathrm{M}$ martensitic transformation, which is practically identical to the critical stress for inducing the martensitic transformation in the solid plate (Fig. 1). By increasing the number of holes from 0 (solid plate) to 3, the flat stress plateaus over $A \leftrightarrow M$ transformations are progressively substituted by stress gradients over stress-induced martensitic transformations. It can be inferred that the perforated plates have better controllability of 
load-displacement over portions of $\mathrm{A} \leftrightarrow \mathrm{M}$ transformations with stress gradients. As observed, the overall transformation strain increases by increase of number of holes.
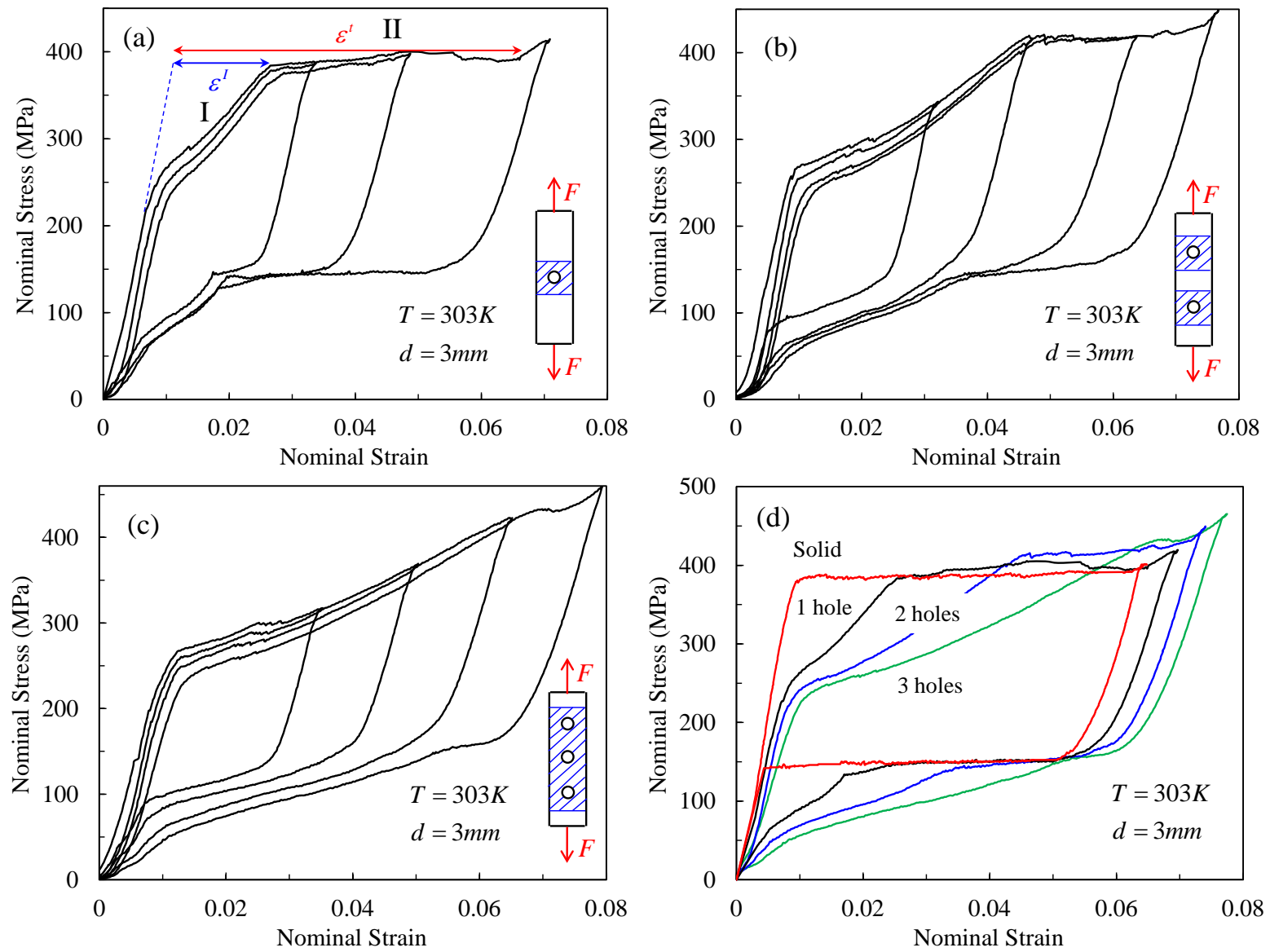

Fig. 2. Deformation behaviour of perforated NiTi plates under tension; (a): one hole, (b): two holes, (c): three holes, (d): comparison of the three samples with solid plate

Fig. 3(a) illustrates the effect of hole size on stress-strain variation of the NiTi plate with one circular hole under uniaxial loading. In comparison with the solid plate, the forward transformation started at a lower loading level (as also seen in Fig. 2). This critical stress for initiating the stress-induced martensitic transformation decreased with increase of hole diameter. Fig. 3(b) shows dependence of the critical stress on hole diameter. The horizontal axis is presented as the ratio of the hole diameter and the plate width. The diagram shows a rather linear decrease of the nominal critical stress with respect to the increase of $d / b$ ratio. Fig. 3(c) depicts the variation of affected length to hole diameter ratio against $d / b$ ratio. It is understood that the ratio of affected length to hole diameter, which is practically $\eta_{s} / \eta_{h}$, remains at a nearly constant value of 3 as $d / b$ ratio varies over studied range. 

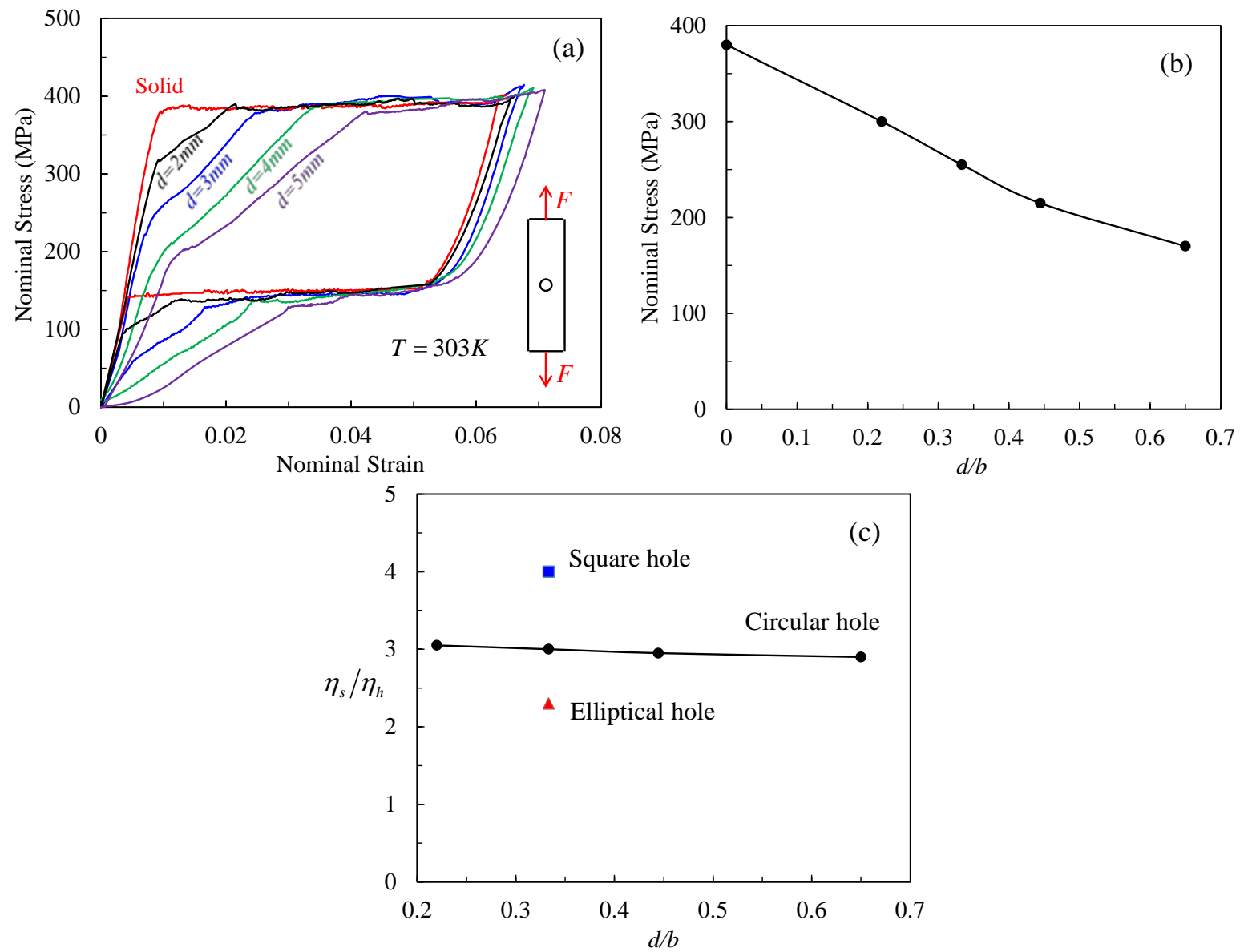

Fig. 3. The effect of hole size on deformation behaviour of perforated NiTi plates under tension; (a): full deformation cycle, (b): critical nominal stress for $\mathrm{A} \rightarrow \mathrm{M}$ transformation, (c): the ratio of affected length to hole diameter (the samples with square and elliptical holes are also shown)

Fig. 4 shows the deformation behaviour of NiTi plates $(9 \mathrm{~mm} \times 35 \mathrm{~mm})$ with non-circular holes, with sample (a) having an elliptical hole and sample (b) having a square hole. The elliptical hole in sample (a) had a minor axis of $3 \mathrm{~mm}$ along the width direction and a major axis of $5 \mathrm{~mm}$ along the length direction of the strip. The square hole in (b) had a side length of $3 \mathrm{~mm}$. It is seen that in both cases the $\mathrm{A} \rightarrow \mathrm{M}$ transformation started at the nominal stress of $\sim 250 \mathrm{MPa}$, as the minimum cross-sectional area is equal for two samples. For the plate with the elliptical hole, the transformation developed over a continuous stress gradient, as expected of the hole geometry. For the plate with the square hole, the transformation firstly evolved over a partial stress plateau relative to the holed region with a constant crosssectional area. Then, the loading level increased progressively to that of the second stress plateau where transformation is mostly completed in the solid part. This deformation behaviour is divided into three regions, as indicated in the figure. Based on the transformation strain fractions of the three regions, the corresponding affected lengths within the gauge length are marked in the sample shown in Fig. 4(b). It is interesting to note that the deformation behaviour exhibited a gradient stress in region II despite that the sample crosssection areas remains unchanged. In Fig. 3(c), the $\eta_{s} / \eta_{h}$ ratios of these samples are demonstrated with square and triangular markers, respectively for the plate with a square hole and the one with an elliptical hole. It is observed that this ratio is higher for the sample with a 
square hole and lower for that with an elliptical hole comparing with a sample with a circular hole.
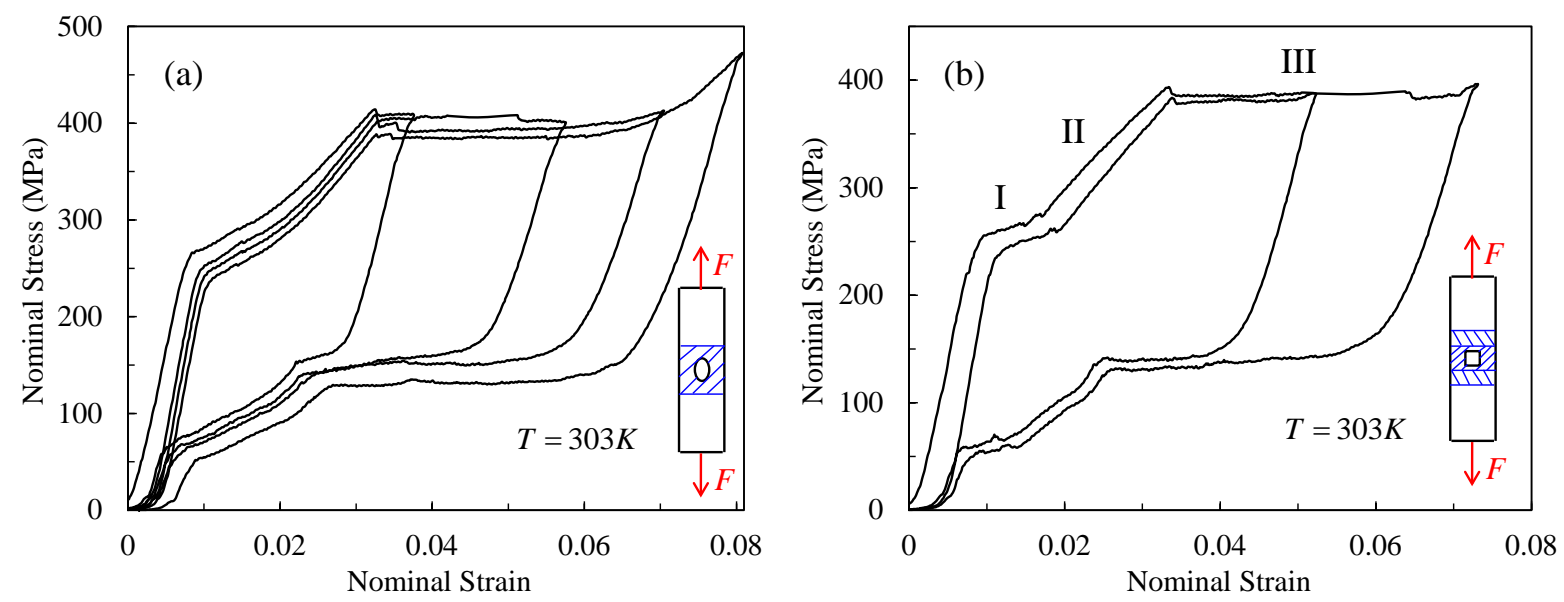

Fig. 4. Deformation behaviour of NiTi plates with a noncircular hole under tension; (a): elliptical hole, (b): square hole

\section{Analytical modelling}

To analytically describe the deformation behaviour of a holed NiTi plate, we use a mathematical approach to obtain the nominal stress-strain curve of such a structure at different stages of loading cycle. Fig. 5(a) shows a NiTi plate of length $L$, width $b$ and thickness $h$ with a circular hole of diameter $d$, positioned symmetrically with respect to $x$ and $y$ axes. We define $\alpha$ as the hole diameter divided by the plate width, i.e. $\alpha=d / b$. The plate is under tensile load $F$ along $x$ axis, uniformly applied over its top and bottoms edges. The forward and reverse transformation stresses and the forward transformation strain (plateau length) of the plate material are denoted as $\sigma_{t}, \sigma_{t}^{\prime}$ and $\varepsilon_{t}$, respectively. The elastic moduli of austenite and martensite phases are $E_{A}$ and $E_{M}$, respectively (all denoted in Fig. 1). To find an analytical solution to the problem, we assume that the stress is uniform within each cross section of the plate along the loading direction. While $0 \leq \sigma<\sigma_{t}(1-\alpha)$, the structure is fully austenite and $\Delta L_{T o t}$ is found as:

$$
\Delta L_{T o t}=2 \int_{0}^{d / 2} \frac{F d x}{E_{A} w(x)}+\frac{F(L-d)}{E_{A} b h}
$$

where $w(x)$ is the width of plate at $x$ in section II marked in Fig. 5(a), and is written as:

$$
w(x)=b-2 \sqrt{\frac{d^{2}}{4}-x^{2}}
$$

Using Eqs. (1), (2) and (3), the nominal stress-strain relation for this stage is found:

$$
\varepsilon=\frac{\sigma b}{E_{A} L}\left(\frac{2}{\sqrt{(1+\alpha)(1-\alpha)}} \tan ^{-1} \sqrt{\frac{1+\alpha}{1-\alpha}}-\frac{\pi}{2}+\frac{L}{b}-\alpha\right)
$$

During $\sigma_{t}(1-\alpha) \leq \sigma<\sigma_{t}$, section II is transformed to martensite with $\mathrm{A} \rightarrow \mathrm{M}$ transformation initiating at the middle and propagating toward the top and bottom ends of this section. 
Variable $x_{A-M}$ expresses the displacement of the A-M boundaries within the plate. It corresponds to the angle variable $\theta$ ranging from 0 to $\pi / 2$ as $x_{A-M}$ varies from 0 to $d / 2$. $\Delta L$ of this stage includes the elongation of austenite and martensite regions as marked in Fig. 5(a) during this stage. Using similar approach reported for geometrically graded $\mathrm{NiTi}$ structures [37, 38], $\Delta L$ during this period can be written as:

$$
\begin{aligned}
& \Delta L=2 \int_{0}^{x_{A-M}} \frac{\left(F-\sigma_{t} w(x)\right) d x}{E_{M} w(x)}+2 \int_{0}^{x_{A-M}} \frac{\left(\sigma_{t} w(x)-\sigma_{t}(b-d) h\right) d x}{E_{A} w(x)}+2 x_{A-M} \varepsilon_{t} \\
& +2 \int_{x_{A-M}}^{d / 2} \frac{\left(F-\sigma_{t}(b-d) h\right) d x}{E_{A} w(x)}+\frac{\left(F-\sigma_{t}(b-d) h\right)(L-d)}{E_{A} b h}
\end{aligned}
$$

Using Eqs. (1), (3) and (5) and considering the strain produced at the end of previous stage (using Eq. (4)), the nominal stress-strain relation of this stage is obtained as a set of two equations:

$$
\begin{aligned}
& \varepsilon=\frac{2 b}{L}\left(\frac{\sigma}{E_{M}}-\frac{\sigma_{t}(1-\alpha)}{E_{A}}\right)\left(\frac{1}{\sqrt{(1+\alpha)(1-\alpha)}} \tan ^{-1}\left(\sqrt{\frac{1+\alpha}{1-\alpha}} \tan \frac{\theta}{2}\right)-\frac{\theta}{2}\right) \\
& +\frac{\alpha b \sin (\theta)}{L}\left(\sigma_{t}\left(\frac{1}{E_{A}}-\frac{1}{E_{M}}\right)+\varepsilon_{t}\right)+\frac{2 b\left(\sigma-\sigma_{t}(1-\alpha)\right)}{E_{A} L} \times \\
& \left(\frac{1}{\sqrt{(1+\alpha)(1-\alpha)}}\left(\tan ^{-1} \sqrt{\frac{1+\alpha}{1-\alpha}}-\tan ^{-1}\left(\sqrt{\frac{1+\alpha}{1-\alpha}} \tan \frac{\theta}{2}\right)\right)+\frac{1}{2}\left(\frac{L}{b}-\alpha+\theta-\frac{\pi}{2}\right)\right) \\
& +\frac{\sigma_{t}(1-\alpha) b}{E_{A} L}\left(\frac{2}{\sqrt{(1+\alpha)(1-\alpha)}} \tan ^{-1} \sqrt{\frac{1+\alpha}{1-\alpha}}-\frac{\pi}{2}+\frac{L}{b}-\alpha\right) \\
& \sigma=\sigma_{t}(1-\alpha \cos \theta)
\end{aligned}
$$

In the above equations, $\sigma$ and $\varepsilon$ are expressed in terms of the common variable $\theta$ changing from 0 to $\pi / 2$.

At $\sigma=\sigma_{t}$, Sections I and III of the plate, denoted in Fig. 5(a), are transformed to martensite, adding $(L-d) \varepsilon_{t}$ to the $\varepsilon$ at the end of transformation of section II. When $\sigma>\sigma_{t}$, the plate is elastically deforming in fully martensite phase. The strain produced during this stage can be obtained from Eq. (4) by substituting $E_{A}$ by $E_{M}$. The unloading stress-strain relations can be established by substituting $\sigma_{t}$ by $\sigma_{t}^{\prime}$ and $\varepsilon_{t}$ by $\varepsilon_{t}-\left(1 / E_{M}-1 / E_{A}\right)\left(\sigma_{t}-\sigma_{t}^{\prime}\right)$ in the above equations and following the procedure of the loading period. 

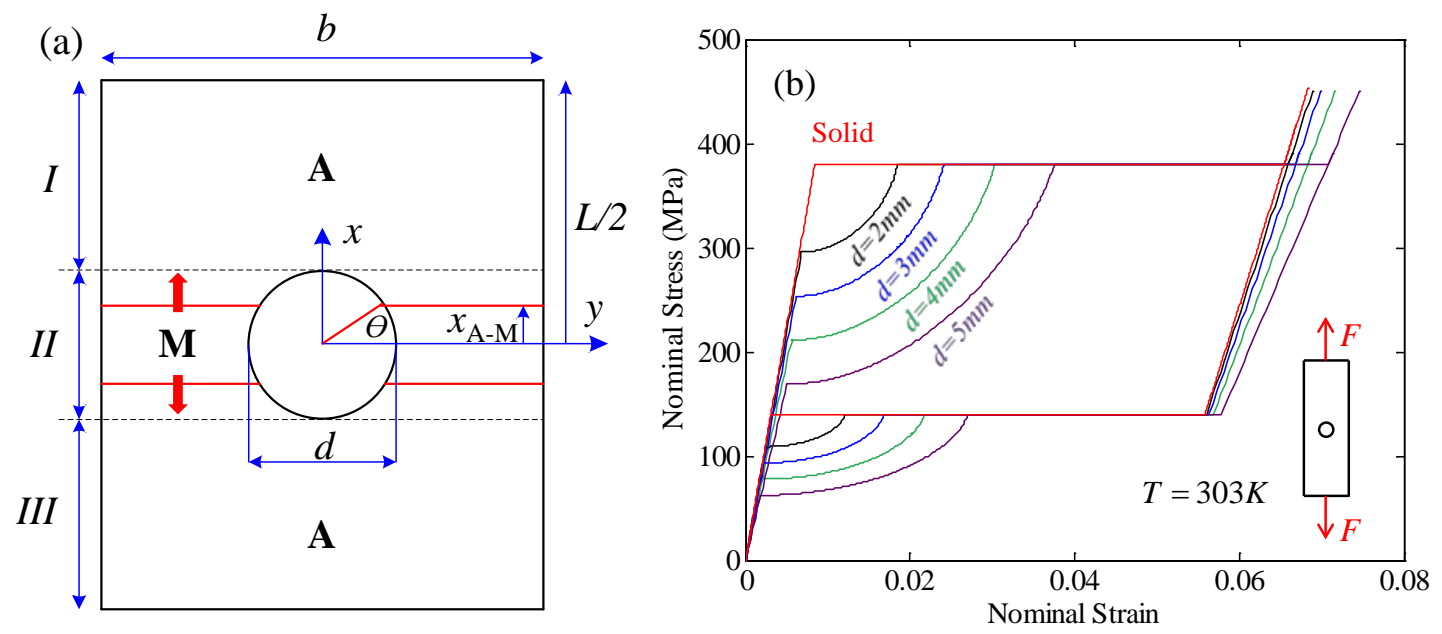

Fig. 5. NiTi plate with a circular hole during martensitic transformation; (a): schematic design, (b): analytical stress-strain diagram

Fig. 5(b) shows the deformation behaviour of the plate based on the developed analytical solution for plates with one hole of different diameters. The material parameters used in the model are obtained from the actual experimental results shown in Fig. 1, as:

$$
\sigma_{t}=380 \mathrm{MPa}, \sigma_{t}^{\prime}=140 \mathrm{MPa}, \varepsilon_{t}=0.057, E_{A}=45 G P a, E_{M}=25 G P a
$$

It is seen that the analytical solution shows of the stress-strain variations exhibit qualitative agreement with the experimental observations shown in Fig. 3(a). As this model is a simple 1D model, it does not take into account the stress concentration near the hole. In this regard, the qualitative agreement is satisfactory as a guide for predicting the deformation behaviour of perforated NiTi plates in uniaxial tension.

\section{Conclusions}

The deformation behaviour of perforated pseudoelastic NiTi plates is investigated through experimental and analytical approaches. The main conclusions of the study may be summarised as following.

(1) The nominal stress-strain curves of perforated pseudoelastic NiTi plates for stressinduced martensitic transformation exhibit a distinctive region with a stress gradient associated with the deformation within the hole-affected section. The gradient stress for the transformation renders the material better controllability for stress-initiated actuation.

(2) The expanse of the affected deformation region with stress gradient is dependent on the geometry and number of holes, and the expanse of affected region is larger than the dimension of the holes in the direction of loading. For the sample geometry tested, $33 \%$ of total hole length for uniformly distributed circular holes is enough to result in a totally gradient deformation for stress-induced martensitic transformation.

\section{Acknowledgement}

We wish to acknowledge the financial support to this work from the Australian Research Council in grant DP140103805 and the French National Research Agency Program in grant N.2010 BLAN 90201.

\section{References}


[1] Y. Liu, G.S. Tan, Effect of deformation by stress-induced martensitic transformation on the transformation behaviour of NiTi, Intermetallics, 8 (2000) 67-75.

[2] K. Otsuka, X. Ren, Recent developments in the research of shape memory alloys, Intermetallics, 7 (1999) 511-528.

[3] G. Tan, Y. Liu, Comparative study of deformation-induced martensite stabilisation via martensite reorientation and stress-induced martensitic transformation in NiTi, Intermetallics, 12 (2004) 373-381.

[4] A.S. Mahmud, H. Yang, S. Tee, G. Rio, Y. Liu, Effect of annealing on deformationinduced martensite stabilisation of NiTi, Intermetallics, 16 (2008) 209-214.

[5] E.A. Williams, G. Shaw, M. Elahinia, Control of an automotive shape memory alloy mirror actuator, Mechatronics, 20 (2010) 527-534.

[6] M. Akhlaghi, R. Mahmudi, M. Nili-Ahmadabadi, Deformation energy of NiTi shape memory wires, Mater. Design, 32 (2011) 1923-1930.

[7] I.Y. Khmelevskaya, E.P. Ryklina, S.D. Prokoshkin, G.A. Markossian, E.P. Tarutta, E.N. Iomdina, A shape memory device for the treatment of high myopia, Mater. Sci. Eng. A, 481482 (2008) 651-653.

[8] M.H. Elahinia, M. Hashemi, M. Tabesh, S.B. Bhaduri, Manufacturing and processing of NiTi implants: A review, Prog. Mater Sci., 57 (2012) 911-946.

[9] X. Wang, Y. Bellouard, J.J. Vlassak, Laser annealing of amorphous NiTi shape memory alloy thin films to locally induce shape memory properties, Acta Mater., 53 (2005) 49554961.

[10] K.P. Mohanchandra, G.P. Carman, TiNi thin film devices, in: S. Miyazaki, Y.Q. Fu, W.M. Huang (Eds.) Thin film shape memory alloys: fundamentals and device applications, Cambridge University Press, 2009, pp. 321-345.

[11] B.S. Shariat, Y. Liu, G. Rio, Analytical modelling of functionally graded NiTi shape memory alloy plates under tensile loading and recovery of deformation upon heating, Acta Mater., (2013).

[12] B.S. Shariat, Y. Liu, G. Rio, Thermomechanical modelling of microstructurally graded shape memory alloys, J. Alloys Compd., 541 (2012) 407-414.

[13] Q. Meng, Y. Liu, H. Yang, B.S. Shariat, T.-h. Nam, Functionally graded NiTi strips prepared by laser surface anneal, Acta Mater., 60 (2012) 1658-1668.

[14] D.S. Grummon, J.A. Shaw, J. Foltz, Fabrication of cellular shape memory alloy materials by reactive eutectic brazing using niobium, Mater. Sci. Eng. A, 438-440 (2006) 1113-1118.

[15] T. Duerig, A. Pelton, D. Stöckel, An overview of nitinol medical applications, Mater. Sci. Eng. A, 273-275 (1999) 149-160.

[16] R.-X. Zhang, Q.-Q. Ni, A. Masuda, T. Yamamura, M. Iwamoto, Vibration characteristics of laminated composite plates with embedded shape memory alloys, Compos. Struct., 74 (2006) 389-398.

[17] B.-Y. Li, L.-J. Rong, Y.-Y. Li, V.E. Gjunter, A recent development in producing porous $\mathrm{Ni}-\mathrm{Ti}$ shape memory alloys, Intermetallics, 8 (2000) 881-884.

[18] G. Chen, P. Cao, G. Wen, N. Edmonds, Y. Li, Using an agar-based binder to produce porous NiTi alloys by metal injection moulding, Intermetallics, 37 (2013) 92-99.

[19] K. Krishnakumar, G. Venkatarathnam, Transient testing of perforated plate matrix heat exchangers, Cryogenics, 43 (2003) 101-109.

[20] Y.Y. Lee, E.W.M. Lee, C.F. Ng, Sound absorption of a finite flexible micro-perforated panel backed by an air cavity, Journal of Sound and Vibration, 287 (2005) 227-243.

[21] G. Gan, S.B. Riffat, Pressure loss characteristics of orifice and perforated plates, Exp. Therm Fluid Sci., 14 (1997) 160-165. 
[22] P.K. Kumar, D.C. Lagoudas, Introduction to shape memory alloys, in: D.C. Lagoudas (Ed.) Shape memory alloys: modeling and engineering applications, Springer, 2008. [23] A. Duval, M. Haboussi, T. Ben Zineb, Modelling of localization and propagation of phase transformation in superelastic SMA by a gradient nonlocal approach, Int. J. Solids Struct., 48 (2011) 1879-1893.

[24] B. Samsam, Y. Liu, G. Rio, Computational modelling of deformation of NiTi plates with circular holes, Mater. Sci. Forum, 654-656 (2010) 2091-2094.

[25] B.S. Shariat, Y. Liu, G. Rio, Finite element computational modelling and experimental investigation of perforated NiTi plates under tension, Mater. Res. Bull., 48 (2013) 5099-5104. [26] P. Zhu, A.P. Stebner, L.C. Brinson, A numerical study of the coupling of elastic and transformation fields in pore arrays in shape memory alloy plates to advance porous structure design and optimization, Smart Mater. Struct., 22 (2013) 094009.

[27] B.S. Shariat, Y. Liu, G. Rio, Numerical modelling of pseudoelastic behaviour of NiTi porous plates, J. Intell. Mater. Syst. Struct., (2013).

[28] B.-Y. Li, L.-J. Rong, Y.-Y. Li, Stress-strain behavior of porous Ni-Ti shape memory intermetallics synthesized from powder sintering, Intermetallics, 8 (2000) 643-646.

[29] C. Greiner, S.M. Oppenheimer, D.C. Dunand, High strength, low stiffness, porous NiTi with superelastic properties, Acta Biomater., 1 (2005) 705-716.

[30] Y. Zhao, M. Taya, Y. Kang, A. Kawasaki, Compression behavior of porous NiTi shape memory alloy, Acta Mater., 53 (2005) 337-343.

[31] Y.P. Zhang, D.S. Li, X.P. Zhang, Gradient porosity and large pore size NiTi shape memory alloys, Scripta Mater., 57 (2007) 1020-1023.

[32] M. Barrabés, P. Sevilla, J.A. Planell, F.J. Gil, Mechanical properties of nickel-titanium foams for reconstructive orthopaedics, Mater. Sci. Eng. C, 28 (2008) 23-27.

[33] Z. Guo, H. Xie, F. Dai, H. Qiang, L. Rong, P. Chen, F. Huang, Compressive behavior of 64\% porosity NiTi alloy: An experimental study, Mater. Sci. Eng. A, 515 (2009) 117-130.

[34] M. Panico, L.C. Brinson, Computational modeling of porous shape memory alloys, Int. J. Solids Struct., 45 (2008) 5613-5626.

[35] J.M. Gere, B.J. Goodno, Mechanics of Materials, Cengage Learning, 2009.

[36] B.S. Shariat, Y. Liu, G. Rio, Hystoelastic deformation behaviour of geometrically graded NiTi shape memory alloys, Mater. Design, 50 (2013) 879-885.

[37] B.S. Shariat, Y. Liu, G. Rio, Modelling and experimental investigation of geometrically graded NiTi shape memory alloys, Smart Mater. Struct., 22 (2013) 025030.

[38] B.S. Shariat, Y. Liu, G. Rio, Mathematical modelling of pseudoelastic behaviour of tapered NiTi bars, J. Alloys Compd., (2012). 